\title{
Research on Distribution Network Development Speed Comprehensive Evaluation Method
}

\author{
Hongfei Liu", a , Dongxiao Niu, ${ }^{1, b}$, Yali Huang ${ }^{1, ~ c}$, Han $\mathrm{Wu}^{1, \mathrm{~d}}$ \\ ${ }^{1}$ School of economics and management, North China Electric Power University, Changping District, \\ Beijing 102206 \\ aemail:345371639@qq.com, bemail:niudx@126.com, cemail:huangyali5210@163.com, \\ demail:351614235@qq.com
}

\begin{abstract}
Keywords: Power grid comprehensive evaluation, Evaluation index system, Comprehensive evaluation method

Abstract. The reasonable and accurate assessment of the development speed of the distribution network is very important for a country's power grid construction and power distribution. The comprehensive evaluation system of distribution network developed proposed in this paper can be applied to evaluate the development speed of distribution network in different regions and different development stages. We got eight factors which can comprehensive evaluate the distribution network development speed by analyzing the influencing factors on the development of distribution network. The evaluation system can be well used in the development speed of distribution network.
\end{abstract}

\section{Introduction}

As an important part of the power grid, distribution network is the link between the power generation, transmission system and the users. The development level of distribution network is linked closely to the overall development level of the region. The development speed of distribution network is the direct response to the development level of the regional power grid and the whole development level of the region ${ }^{[1]}$. So the accurate evaluation of the development speed of distribution network is of great significance. It not only can provide the basis for the measurement of regional power grid development potential, but also can provide an important reference for the measurement of regional economic and social development potential.

At present, the research on evaluation of distribution network is usually about the power supply reliability, economy, security, power supply quality and other characteristics, which evaluates the technical level of distribution network from different aspects. Reference [2] set up a comprehensive evaluation index system for distribution network from the technical rationality and security and gave the evaluation method by examples. Reference [3] classified the index according to the characteristics of index attribute and then obtained the content of the evaluation index system of distribution network. Reference [4] carried out the quantitative evaluation of the distribution network from network structure, technical level of equipment by adopting AHP and Delphi. The optimization principle and grey correlation degree analysis were used to analyze the construction scale of the high voltage distribution network in Reference [5] and [6]. The results of these studies always focus on the research of the traditional technical indicators, paying attention to the internal cause of distribution network development. That is to say, these research from the point of view of the electric power enterprise, mainly reflects the distribution network operation ability and is not comprehensive for the assessment on the whole ability of the distribution network, which can't provide intuitive and deep reaction on the distribution network actual development situation and development potential. Besides, with the arrival of the era of large power grid, no matter from the angle of the construction of power grid or the development of the power grid, it is not right to analyze the grid development from the internal factors of the power grid development. The external factors affecting the power grid development have become the important factors that can't be ignored ${ }^{[7]}$.

This paper made a deep analysis on the internal and the external factors of the development of distribution network and built a comprehensive evaluation index system of the development rate of the 
whole distribution network which was suitable for distribution networks of different regions and different stages. It will contribute to the analysis of power grid planning and measures and can provide a theoretical basis for the distribution network construction and transformation project and provide the basis for measuring the distribution network development potential and the local social development potential.

\section{Evaluation index system of distribution network development speed}

\section{Index selection}

In this paper, the key is to construct evaluation index system of the distribution network development speed. Before selecting the indexes, we should first analyze the characteristics and driving factors of the distribution network, so as to provide a basis for selection evaluation index of distribution network development speed.

Distribution network is dispersed in many spots, long lines and quite wide areas. It is the end of the network and closely linked with the users. There are many factors influencing the distribution network and they are much different in different regions. These characteristics of distribution network must be taken into consideration when constructing the evaluation index system of distribution network development speed to ensure the practicability and operability of evaluation index system. At the same time, we must make sure the data sources are true and reliable to ensure the assessment results objective and impartial. In addition, the aim of constructing the distribution network development speed evaluation system is to measure the development potential of the distribution network and provides the basis for the distribution network planning. Therefore, it is necessary to consider the influence of power users, society and electric power enterprises in the development of distribution network.

By the analysis of the features of distribution network and power users, power enterprises and the society, eight influence factors of distribution network development level can be drawn: grid coordinate adaptability, power structure and power supply capacity, power supply efficiency, reliability, electric energy quality, equipment level, management level and the operation efficiency. When the distribution network meet the requirements from the above 8 aspects, it can be considered that the development level of the distribution network project is in a good level.

The factors of social influence are taken as an example to illustrate the method by using the fishbone analysis to look for the two level indexes. Fishbone diagram analysis method, also known as causal analysis method, is a convenient and reliable method by interrelated the relationship between the characteristics and influence factors into logical graphics with the important factors marked. The factors of social effects by adopting fishbone diagram analysis are shown in figure $1^{[8]}$.

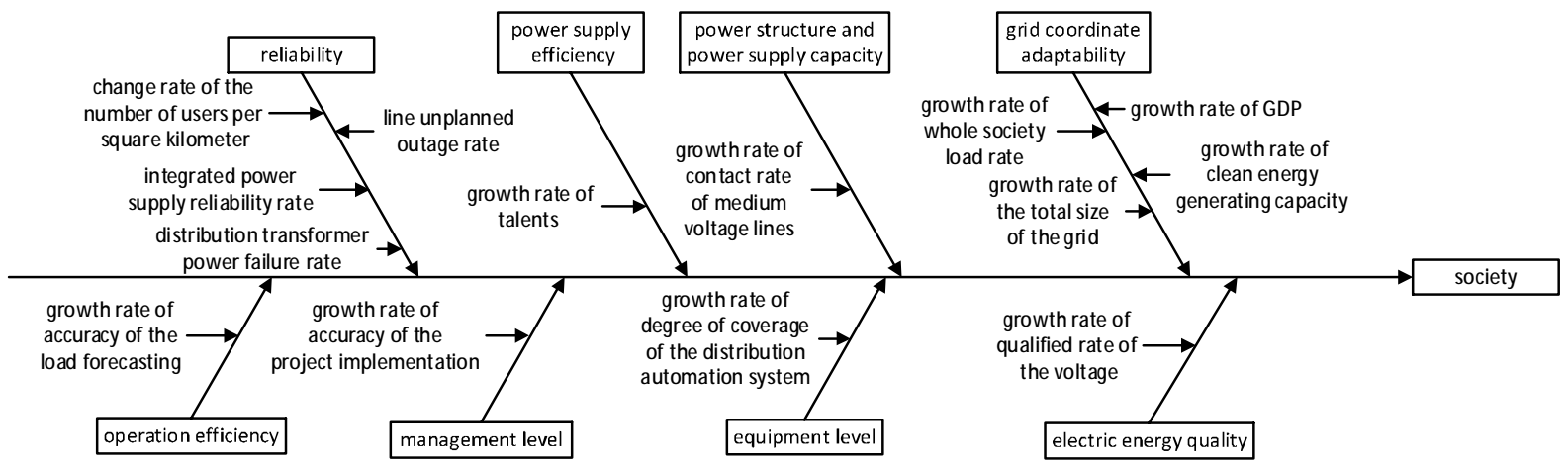

Figure.1 Influence factors in society

The influence factors in society from grid coordinate adaptability include growth rate of GDP, growth rate of whole society load rate, growth rate of clean energy generating capacity, growth rate of the total size of the grid. The influence factor in society from power structure and power supply capacity includes growth rate of contact rate of medium voltage lines. The influence factor in society from power supply efficiency includes growth rate of talents. The influence factors in society from 
reliability include change rate of the number of users per square kilometer, integrated power supply reliability rate, distribution transformer power failure rate, line unplanned outage rate. The influence factor in society from electric energy quality includes growth rate of qualified rate of the voltage. The influence factor in society from equipment level includes growth rate of degree of coverage of the distribution automation system. The influence factors in society from management level and the operation efficiency include growth rate of accuracy of the load forecasting, growth rate of accuracy of the project implementation.

The same method was used to analyze the impact factors of power users and power companies and the same index had been merged to get the selection of evaluation indexes of distribution network development. There are 31 indexes in total.

\section{Establishment of hierarchical structure system}

The hierarchical structure of the comprehensive evaluation index system of distribution network development level is shown in figure 3. From the figure, grid coordinate adaptability (A), power structure and power supply capacity (B), power supply efficiency (C), reliability (D), electric energy quality $(E)$, equipment level $(F)$, management level $(G)$ and the operation efficiency $(H)$ are the first level attributes. Growth rate of whole society load rate (A1), growth rate of the whole social load rate (A2), growth rate of clean energy generating capacity (A3) and so on are the second level attributes. The comprehensive evaluation index system of distribution network development level is shown in figure 2 .

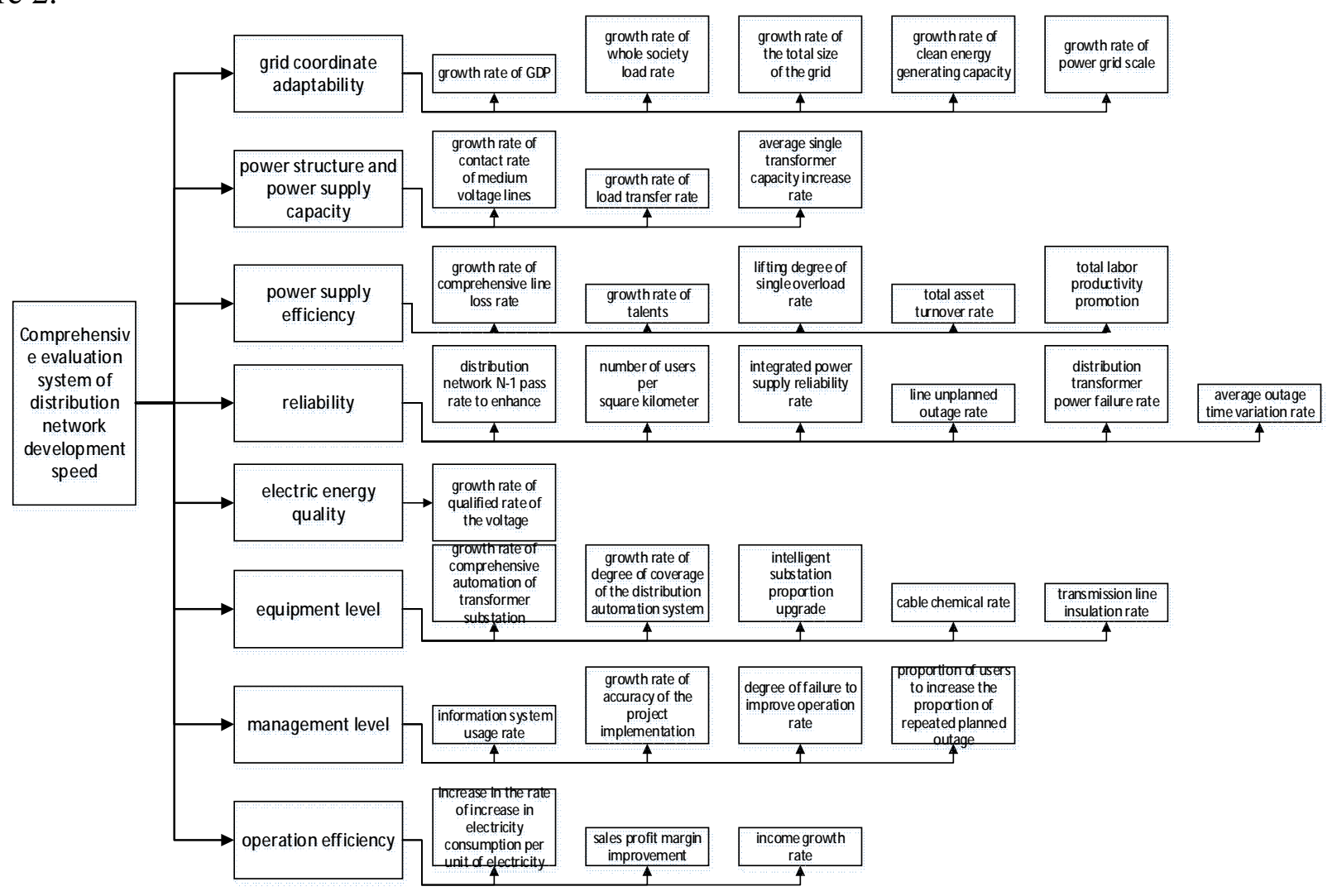

Figure.2 Comprehensive evaluation system of distribution network development speed

The index system proposed in this paper includes 3 layers, which is clear and simple. It can comprehensively and objectively evaluate the development speed of distribution network with good integrity, practicality and maneuverability. The underlying indicators have clear meaning and the data needed to calculate is easy to access. 


\section{Grading standards and index weight}

\section{Grading standards}

(1) index calculation method

The calculation method of evaluation index of the development level of distribution network is mainly used to evaluate the development level of distribution network. By determining the calculation method of evaluation index, the evaluation index is quantified to realize the evaluation of the development level of distribution network. In order to realize the horizontal comparison between the different regions, different stages of development of distribution network, the indexes in the system were calculated with its change rate to achieve no quantization with percentage form finally. In this way, the calculation method for the index is as follows.

$$
x_{i}=\left(x_{i p}-x_{i l}\right) / x_{i l} \times 100 \%, i=1,2, \cdots, 31
$$

Where, ${ }^{x_{i}}$ is the value of the index i. ${ }^{x_{i p}}$ is the present value of the index $i$ and ${ }^{x_{i l}}$ is the last value of the index $i$.

(2) index system scoring criteria

Scoring criteria is mainly made according to the type and the ideal value of the index. Indicators are divided into the monotonic indicators and non-monotonic indicators. Among them, the monotonic indicators are divided into positive indicators and negative indicators. For the positive indicators, the higher the index value, the higher the score. For the negative indicators, the smaller the index value, the higher the score. For the non-monotonic indicators, the score will be the highest at a certain point and be smaller when far away from the point.

From the calculation method of each index, the index values of distribution network development level evaluation system are shown as percentage. And no matter the index value is positive or negative, the higher the index value, the higher the score after calculating the growth rate of the index. Therefore, in order to be able to set up the evaluation criteria of each evaluation index, we assumed that the corresponding relationship between the index value and the score is a linear relation.

$$
x_{i}^{\prime}=100 \times x_{i}, i=1,2, \cdots, 31
$$

Where, $x_{i}^{\prime}$ is the score of the index $i$.

\section{Index weight}

(1) analytic hierarchy process

Analytic hierarchy process (AHP), is a systematic analytical method with qualitative analysis and quantitative analysis. According to the logic of the subordinate relationship, the analytic hierarchy process divides all the influencing factors into a series of hierarchical structure. The weights of the lowest level of the factors are got based on mathematical method and the weights of the last layer are obtained. The weight of each layer can be got by this method. AHP has the advantages of high reliability and is widely used in all kinds of evaluation method. The steps of AHP can be summarized as follows.

1) Establish the hierarchical structure model according to the actual situation after deeply analyzing the problems considered.

2) Construct the judgment matrix according to the relative importance of the elements in the hierarchy mechanism model.

3) Calculate the weights after checking the consistency of the judgment matrix.

4) Calculate the comprehensive evaluation value and rank the evaluation objects.

(2) index weight

The calculation of index weights is an important part in the process of evaluation, which has a direct impact on the evaluation results. we use the method of analytic hierarchy process to get the weights in this paper. We determined the weight of each index by comprehensive utilization of expert knowledge and experience. For intuitive and convenient calculation, the approximate value of the weight is taken. And the sum of the weights of the same layer is 1 . Each index weight is shown in table 1. 
Table.1 Distribution network development speed evaluation index weight

\begin{tabular}{|c|c|c|c|}
\hline Layer 1 & Layer 2 & Layer 3 & weight \\
\hline \multirow{32}{*}{$\begin{array}{l}\text { Distribution } \\
\text { network } \\
\text { development } \\
\text { speed } \\
\text { evaluation } \\
\text { system }\end{array}$} & \multirow{6}{*}{$\begin{array}{l}\text { grid coordinate } \\
\text { adaptability } \\
(0.1990)\end{array}$} & growth rate of GDP & 0.0685 \\
\hline & & growth rate of whole society load rate & 0.032 \\
\hline & & growth rate of power grid scale & 0.0135 \\
\hline & & growth rate of the total size of the grid & 0.0165 \\
\hline & & growth rate of clean energy generating capacity & 0.0685 \\
\hline & & growth rate of contact rate of medium voltage lines & 0.0314 \\
\hline & \multirow{2}{*}{$\begin{array}{l}\text { power structure and } \\
\text { power supply } \\
\text { capacity } \\
(0.0493)\end{array}$} & growth rate of load transfer rate & 0.0127 \\
\hline & & average single transformer capacity increase rate & 0.0052 \\
\hline & \multirow{5}{*}{$\begin{array}{l}\text { power supply } \\
\text { efficiency } \\
(0.1990)\end{array}$} & growth rate of comprehensive line loss rate & 0.1139 \\
\hline & & growth rate of talents & 0.016 \\
\hline & & lifting degree of single overload rate & 0.0265 \\
\hline & & total asset turnover rate & 0.0213 \\
\hline & & total labor productivity promotion & 0.0213 \\
\hline & \multirow{6}{*}{$\begin{array}{l}\text { reliability } \\
(0.2121)\end{array}$} & distribution network $\mathrm{N}-1$ pass rate to enhance & 0.0891 \\
\hline & & number of users per square kilometer & 0.0077 \\
\hline & & integrated power supply reliability rate & 0.041 \\
\hline & & distribution transformer power failure rate & 0.0166 \\
\hline & & line unplanned outage rate & 0.041 \\
\hline & & average outage time variation rate & 0.016 \\
\hline & \multirow[t]{2}{*}{$\begin{array}{c}\text { electric energy } \\
\text { quality } \\
(0.0319)\end{array}$} & growth rate of qualified rate of the voltage & 0.0319 \\
\hline & & $\begin{array}{l}\text { growth rate of comprehensive automation of } \\
\text { transformer substation }\end{array}$ & 0.0717 \\
\hline & \multirow{4}{*}{$\begin{array}{l}\text { equipment level } \\
\qquad(0.199)\end{array}$} & $\begin{array}{l}\text { growth rate of degree of coverage of the distribution } \\
\text { automation system }\end{array}$ & 0.0717 \\
\hline & & intelligent substation proportion upgrade & 0.0302 \\
\hline & & cable chemical rate & 0.0127 \\
\hline & & transmission line insulation rate & 0.0127 \\
\hline & \multirow{4}{*}{$\begin{array}{c}\text { management level } \\
(0.0488)\end{array}$} & information system usage rate & 0.0061 \\
\hline & & $\begin{array}{l}\text { growth rate of accuracy of the project } \\
\text { implementation }\end{array}$ & 0.0061 \\
\hline & & degree of failure to improve operation rate & 0.0183 \\
\hline & & $\begin{array}{l}\text { proportion of users to increase the proportion of } \\
\text { repeated planned outage }\end{array}$ & 0.0183 \\
\hline & \multirow{3}{*}{$\begin{array}{l}\text { operation efficiency } \\
\qquad(0.0608)\end{array}$} & $\begin{array}{l}\text { increase in the rate of increase in electricity } \\
\text { consumption per unit of electricity }\end{array}$ & 0.0055 \\
\hline & & sales profit margin improvement & 0.0276 \\
\hline & & income growth rate & 0.0276 \\
\hline
\end{tabular}

From table 1, network reliability holds the most important position in the evaluation of distribution network development level and has an important influence to the whole evaluation system. Grid coordinate adaptability, equipment level and power supply efficiency have a sub-important position in the evaluation of distribution network development level. However power structure and power supply capacity, electric energy quality, management level and the operation efficiency are much less important than other four indexes. 


\section{Conclusion}

The comprehensive evaluation system of distribution network developed proposed in this paper can be applied to evaluate the development speed of distribution network in different regions and different development stages. We got eight factors which can comprehensive evaluate the distribution network development speed by analyzing the influencing factors on the development of distribution network. The indexes include grid coordinate adaptability, power structure and power supply capacity, power supply efficiency, reliability, electric energy quality, equipment level, management level and the operation efficiency. According to these 8 factors and its refinement, we can make the refinement accurate evaluation on the development speed of distribution network. The evaluation system can be well used in the development of distribution network.

\section{Acknowledgements}

This work was financially supported by the engineering project of State Grid Zhejiang Electric Power Company named study on the adaptive weight system of the power grid evaluation indexes.

\section{References}

[1] Wang Chengshan, Luo Fengzhang. Comprehensive evaluation theory and method of power distribution system[M]. Beijing: Science Press, 2012.

[2] Xiao Jun, Gao Haixia, Ge Shaoyun, et al. Urban medium voltage distribution network evaluation method and case study[J]. Power system technology, 2005, 29 (2): 77-81.

[3] Cui Kai, Shi Zinan, Zhao Juan. Study on evaluation index system of distribution network[J]. Electric power construction, 2013, 34 (2): 18-21.

[4] Feng Xinlong, Sun Yan, et al. A comprehensive evaluation index system and evaluation method for distribution network[J]. Guangdong electric power, 2013, 26 (11): 20-25.

[5] Li Xinran, Li Peiqiang, Zhu Xiangyou, et al. Evaluation of the construction scale of UHV power distribution network based on optimization theory[J]. Automation of electric power systems, 2007, 31 (4): 46-50.

[6] Li Xinran, Liu Yanghua, Zhu Xiangyou, et al. Evaluation index system and Application Research on the construction scale of high voltage distribution network[J]. Proceedings of the Chinese society of electrical engineering, 2006, 26 (17): 18-24.

[7] Liu Lianguang, Chen Wei, Liu Zifa, et al. Evaluation problems and evaluation system for the adaptability of large power network[J]. Electric power construction, 2015, 36 (10): 138-143.

[8] Zhao Lei, Qin Jin Gou, et al. Evaluation index system of distribution network project[J]. Power grid clean energy, 2014,30 (6): 19-23. 